\title{
Avaliando o impacto dos rendimentos de aposentadoria no mercado de trabalho brasileiro
}

\author{
Valdecir Teófilo Moreno ${ }^{1}$ \\ Paulo Aguiar do Monte $^{2}$
}

\begin{abstract}
Resumo: Esse trabalho procura avaliar o impacto dos rendimentos da aposentadoria no mercado de trabalho brasileiro. Para isso, comparou-se o valor da mão de obra, medido em termos salariais, de dois grupos de trabalhadores: grupo 1 , formado por trabalhadores que recebem algum rendimento de aposentadoria e que exercem algum tipo de atividade econômica; grupo 2, formado por trabalhadores que exercem algum tipo de atividade econômica, mas que não recebem rendimento de aposentadoria. Com dados da Pesquisa Nacional de Amostras por Domicilio (PNAD), 2011, e fazendo uso da metodologia Propensity Score Matching (PSM) e da análise do Averge Treatment Effect on the Treated (ATT) foi possível inferir que os trabalhadores aposentados que estão ocupados no mercado de trabalho aceitam uma oferta de emprego com salário inferior, comparativamente, ao salário recebido pelo trabalhador não aposentado (cerca de $58 \%$ menos). Por fim, a robustez do modelo foi realizada através do teste de Rosenbaum.
\end{abstract}

Palavras-chave: Idosos; Aposentadoria; Mercado de trabalho.

JEL: J31, J26, J14.

1 Mestrando em Economia Aplicada na Universidade Federal da Paraíba (UFPB).

Email: prof.valdecir@gmail.com

2 Doutor em Economia. Professor da Universidade Federal da Paraíba (UFPB).

Email: pauloaguiardomonte@gmail.com 


\title{
Evaluating the impact of retirement benefits in the Brazilian labor market
}

\begin{abstract}
This paper evaluates the impact of retirement income in the Brazilian labor market. In order to do this, we compared the wages of two groups of workers: group 1, formed by workers who receive retirement income and who are employed in the labor market; group 2, formed by workers who are employed in the labor market but not receiving retirement income. Based on data from the National Household Sample Survey (PNAD), 2011, and using the Propensity Score Matching (PSM) methodology and the analysis of Averge Treatment Effect on the Treated (ATT) it was possible to infer that retired workers who are regularly employed in labor market accept a job offer with lower salary compared to the salary received by the non-retired workers (around 58\% less). Finally, in order to confirm the robustness of the model, we performed the test of Rosenbaum.
\end{abstract}

Keywords: Elderly; Retirement; The labour market.

JEL: J31, J26, J14.

\section{Introdução}

Até a década de 80 o Brasil era considerado um país de muitos jovens, formando uma pirâmide bem definida, com base larga e topo fino. No entanto, conforme os dados recentes do Censo Demográfico de 2010 (IBGE 2012), a pirâmide etária mostrou uma alteração no seu formato (BIRD 2011), engrossando o tronco e afinando a base, resultado do envelhecimento da população ${ }^{1}$. Este aumento dos anos de vida pode ser justificado por alguns fatores, dentre os quais, os benefícios proporcionados pelos avanços da medicina, em especial os que resultaram na redução das taxas de mortalidade e de fecundidade, e a maior cobertura dos programas sociais e dos programas previdenciários, o que tem levado a uma reestruturação do mercado de trabalho, dividindo-o em segmentos cada vez mais consolidados. (Wajnman et al. 2004; Liberato 2003; Pérez et al. 2005; Kon 2006).

A mudança demográfica e seus impactos no mercado de trabalho tem sido alvo de pesquisas no Brasil. Todavia, especificamente sobre a população idosa no mercado de trabalho brasileiro ainda são poucos os estudos sobre o tema que, de uma forma geral, têm se concentrado na questão da ampliação da oferta de trabalho, com destaque para os trabalhadores aposentados e seus determinantes salariais.

No referente à ampliação da oferta de mão de obra com a permanência (ou retorno) dos trabalhadores aposentados ao mercado de trabalho, Liberato 1 Embora este fenômeno seja mundial, nos países desenvolvidos esse processo ocorreu de forma mais lenta ao longo dos anos, enquanto na América Latina têm ocorrido com taxas de crescimento elevadíssimas (Truelsen et al., 2001). 
(2003) destaca o aumento de $25 \%$ para $33 \%$ da taxa de atividade dos homens aposentados, com idade entre 40 e 80 anos, durante as décadas de oitenta e noventa. Queiroz et al. (2012) destacam que a média de atividade no período de 1995 a 2099 foi de 29,4\% e 35,1\% para aposentados e não aposentados, respectivamente. Já no que respeita o rendimento do trabalhador, Liberato (2003), ao relacionar a força de trabalho pós-aposentadoria aos níveis de escolaridade, idade e valor do benefício recebido, concluiu que os trabalhadores que se mantem no emprego depois da aposentadoria são mais jovens e com menor valor de benefícios, sugerindo que dentre as razões que o levam a continuar trabalhando mesmo depois de aposentado está a necessidade de complementação de renda sobre o valor da aposentadoria. Carrera-Fernandez e Menezes (2001) reforça que a inserção dos idosos no mercado de trabalho está relacionada à renda do trabalho principal e a outras rendas não vinculadas ao trabalho. Blau (1994) destaca que parte dos trabalhadores aposentados opta por reduzir as horas trabalhadas e permanecer no mercado de trabalho por mais tempo.

Neste contexto, o objetivo do artigo é analisar o impacto dos rendimentos de aposentadoria no salário dos trabalhadores no mercado de trabalho brasileiro. Assim, o trabalho se propõe a comparar dois grupos de trabalhadores que contém características observáveis comuns, sendo, um grupo formado por trabalhadores que recebem algum rendimento de aposentadoria e que exercem algum tipo de atividade econômica (Grupo 1 - Grupo Tratado) e o outro grupo formado por trabalhadores que possuem as mesmas características observáveis e que exercem algum tipo de atividade econômica, mas que não recebem rendimento de aposentadoria (Grupo 2 - Grupo Controle). A amostra será restrita aos indivíduos com idade entre 60 e 80 anos $^{2}$.

A importância deste estudo baseia-se na discussão acerca do salário de reserva dos trabalhadores idosos que possuem um rendimento adicional, no caso, o benefício da aposentadoria. Dado que, segundo a Teoria Neoclássica da oferta de mão de obra, haverá um nível de salário abaixo do qual o trabalhador não participará do mercado de trabalho, seria factível supor que, quanto maior for o montante da renda oriunda do não trabalho (por exemplo, o benefício da aposentadoria) maior seria o seu salário de reserva. Por outro lado, a necessidade de complementação de uma renda mínima necessária a sobrevivência do trabalhador, poderá leva-lo a aceitar ofertas salariais mais baixas ${ }^{3}$.

Além desta introdução, o trabalho encontra-se dividido em mais quatro seções. A seção 2 reporta-se a análise do perfil da população idosa no Brasil. A seção 3 refere-se ao procedimento metodológico adotado, especificamente, ao uso da metodologia Propensity Score Matching (PSM) e da determinação

2 A escolha baseia-se na atual legislação brasileira que permite ao trabalhador do sexo feminino solicitar sua aposentadoria a partir dos 60 anos. Ademais, buscou-se uma faixa de idade capaz de tornar os grupos populacionais o mais próximo possível.

3 Cabe ressaltar que a discussão sobre as variáveis que interferem no salário de reserva é bastante complexa, ultrapassando os limites das discussões teóricas deste estudo. Para maiores detalhes ver Bojas (2012) e Pencavel (1986). 
do Average Treatment Effect on the Treated (ATT), além da base de dados utilizada. A seção 4 é destinada as discussões dos resultados. Por fim, a seção 5 refere-se às considerações finais.

\section{O idoso no mercado de trabalho brasileiro e a Previdência Social}

A preocupação social com o envelhecimento da população está presente nos vários debates na forma de fóruns e encontros nacionais que começaram a acontecer mais efetivamente a partir a década de 90. Dados da Organização Mundial da Saúde (OMS) revelam que em 2025 o Brasil será o sexto país do mundo, em número de idosos (OPAS 2005). Nos últimos anos vários trabalhos foram realizados, principalmente envolvendo questões relacionadas à Previdência Social4.

Em 1991, o Governo Federal instituiu o Plano de Benefícios da Previdência Social, alterando algumas regras da aposentadoria, dentre as quais o valor do benefício e a permissão da aposentadoria do trabalhador sem o desligamento ou afastamento do local do trabalho. Para Furtado (2005) e Gasparini et al. (2007), as ações do governo são responsáveis pela alteração observada na proporção de idosos ativos a partir da década de 90. Mais recentemente, em 2012, o total de pessoas economicamente ativas, com mais de 60 anos, representava cerca de 4,0\% da força de trabalho contra 3,45 em 2001.

A inserção do idoso no mercado de trabalho é uma tendência mundial. Segundo Queiroz e Ramalho (2009), os índices de participação são crescentes e apontam para cerca de 1,9 bilhão de idosos no mundo em 2050, podendo chegar a um terço da população nos países mais desenvolvidos. O último Censo Demográfico (IBGE 2012), no referente à expectativa de vida do brasileiro, mantém os índices de envelhecimento em constante crescimento, de modo que, nos últimos noventa anos a expectativa de vida do brasileiro mais do que dobrou, chegando em 2013 a 73,1 anos. Tal evolução repercute diretamente no mercado de trabalho visto que o envelhecer com saúde permite que o trabalhador chegue ao tempo da aposentadoria com disposição para continuar produtivo e ativo no mercado de trabalho. Esta disposição é justificada como um dos motivos que explicariam o retorno do aposentado ao mercado de trabalho e/ou a sua permanência mesmo após a aposentadoria.

O aumento da esperança de vida do brasileiro obrigou o Governo a realizar alterações nos planos de aposentadoria da previdência social a fim de evitar que o sistema entrasse em colapso. A Constituição de 1988 foi responsável 4 Pode-se citar a Universidade de São Paulo-USP, através de da Universidade Aberta à Terceira Idade-UATI, a Universidade de Brasília-UNB, através do Núcleo de Estudos e Pesquisas da Terceira Idade-NEPTI, a Universidade Federal de Sergipe através do Núcleo de Pesquisas e Ações da Terceira Idade-NUPATI; Universidade Federal do Estado de São Paulo-UNIFESP, Universidade Metodista do Estado de São Paulo- UMESP, Pontifícia Universidade Católica de São Paulo- PUCSP, Universidade Presbiteriana Makenzie, dentre outras. 
pela equiparação dos benefícios (rural e urbano) e pelo piso unificado igual a um salário mínimo para todos os benefícios previdenciários e assistenciais. Em seguida, vieram várias reformas da Previdência com o objetivo de suportar as novas demandas financeiras que surgiram com a referida Constituição. Anteriormente, o salário da aposentadoria era composto apenas pela média dos últimos 36 salários de contribuição ${ }^{5}$ ao Instituto Nacional da Seguridade Social - INSS. Após várias reformas nas regras de benefícios da previdência, foi criado o fator previdenciário, baseado na expectativa de vida e na média sobre os valores dos últimos 180 salários de contribuição ${ }^{6}$. Os benefícios previdenciários do Regime Geral da Previdência Social (RGPS) são distribuídos em duas categorias: previdenciários e acidentais. De acordo com o boletim estatístico do Instituto Nacional de Seguridade Social (INSS, 2013), as aposentadorias representam 25,35\% dos benefícios previdenciários do RGPS, destes, cerca de 14,18\% são destinados para aposentadorias por idade, 6,90\% para aposentadorias por tempo de contribuição e 4,27\% para aposentadorias por invalidez. O perfil demográfico dos aposentados, segundo o boletim do INSS (2013), é formado em sua maioria por residentes da zona urbana (54\%), cujo valor da renda média para os aposentados por idade se situa entre $\mathrm{R} \$ 928,52$, na zona urbana, e R \$679,57, na zona rural. Já em relação aos aposentados por tempo de contribuição, o valor da renda média na zona urbana é de $\mathrm{R} \$$ $1.604,84$ e de $\mathrm{R} \$ 801,71$ na zona rural 7 .

Estes rendimentos, apesar de bastante díspares conforme a zona de residência (urbana ou rural) são fundamentais para minimizar os efeitos da desigualdade de renda, além se servir de fonte de renda para parcela considerável da população. Estudos sobre o impacto dos rendimentos de aposentadoria no mercado de trabalho têm constatado que as aposentadorias e pensões cumprem um importante papel no rendimento das famílias mais pobres e das áreas rurais, contribuindo principalmente para a redução da pobreza nas regiões mais precárias do país (Maia et al. 2008). Outra questão importante é destacada por Hoffmann (2010) que argumenta que o rendimento de aposentadorias e pensões também contribui para aumentar a escolarização dos indivíduos jovens, postergando sua inserção no mercado de trabalho.

\section{Procedimento Metodológico}

\subsection{Propentisy Score Matching}

Como procedimento metodológico utilizou-se a técnica de pareamento e probabilidade denominada Propensity Score Matching (PSM). O Propensity

5 O tempo de contribuição era de 30 anos para as mulheres e de 35 para os homens 6 Para maiores informações consulte: Lei no 8.213, de 24 de julho de 1991 e alterações posteriores; Lei n ${ }^{0} 10.666$, de 8 de maio de 2003 e alterações posteriores; Decreto $n^{0}$ 3.048, de 6 de maio 1999 e alterações posteriores; Instrução Normativa INSS/PRES n ${ }^{\circ} 45$, de 06 de agosto de 2010.

7 Boletim Estatístico do INSS de Março de 2013. 
Score Matching é considerado um dos mais eficientes métodos de pareamento utilizado para comparar dois grupos de indivíduos com características observáveis comuns, sendo já bastante difundido na literatura ${ }^{8}$. De uma forma geral, o PSM é utilizado para medir e avaliar o impacto de medidas sociais, experimentos na área de medicina e em testes de efeitos de medicamentos ${ }^{9}$.

Para a formação dos grupos a serem analisados é fundamental que sejam selecionados indivíduos com características semelhantes. Primeiramente tem-se que atentar para o fato de que nem todas as características de uma pessoa podem ser observadas visto que existem aspectos individuais que não são observados, como a determinação e o desejo. A comparação de características observáveis ou elementos observáveis fornece um padrão chamado de método contra factual, que dependerá do maior número possível de características observáveis entre dois indivíduos.

O propensity score é definido por Rosembaum e Rubin (1983) como a probabilidade condicional de um grupo receber o tratamento dado às diversas características em comum. Considerando, inicialmente, a seguinte função probabilidade:

$$
p(x)=P(D=1 \mid X)=E(D \mid X)
$$

Onde, $D=\{0,1\}$ é um indicador de exposição a um tratamento e $X$ é um vetor multidimensional de características do tratado. É possível demonstrar que, se a exposição ao tratamento for aleatória dentro de células definidas por $X$, ela também é aleatória dentro das células definidas pelos valores de uma variável unidimensional $p(x)^{10}$.

Em seguida, definindo-se a variável $Y_{i}\left(D_{i}\right)$ como sendo os resultados potenciais nas duas situações de tratamento, de modo que $Y_{i}(1)$ é o valor da variável resultado para o individuo i sujeito ao tratamento e $Y_{i}(0)$ é o valor da variável resultado para o indivíduo i não sujeito ao tratamento, tem-se uma expressão geral para a variável resultado que pode ser expressa como:

$$
Y_{i}=D_{i}\left(Y_{1}(1)\right)+\left(1-D_{i}\right) Y_{i}(0)
$$

Assim, o efeito causal do tratamento para o indivíduo $i$ pode ser escrito como:

$$
\Delta_{i}=Y_{i}(1)-Y_{i}(0)
$$

O segundo valor do lado direito da expressão não pode ser calculado pois o mesmo não é observado dado que é decorrente do valor da variável resultado para o individuo $i$ caso ele não tivesse sido submetido ao tratamento. Se o propensity score $p(x)$ é conhecido, então o efeito médio do tratamento so- 
bre os tratados - Average Treatment Effect on the Treated (ATT) - pode ser observado como resultado das probabilidades de tal fenômeno.

Após a estimação do ATT, será feita a análise de sensibilidade em ATT para verificar o grau de robustez do modelo. A análise de sensibilidade calcula o "quanto" de viés de seleção poderá estar presente a fim de tornar aceitável a hipótese nula de que não há efeito de tratamento ${ }^{11}$. A análise é feita com base em três pontos: (a) a magnitude do valor estimado para o ATT; (b) seu sinal e (c) sua significância estatística. Se os resultados obtidos com a nova especificação forem similares aos resultados alcançados através do modelo original então, pode-se considerar que as estimativas são robustas a pequenas mudanças na especificação do modelo.

Segundo Dehejia (2005), caso os resultados sejam altamente sensíveis a mudanças na especificação, então tal método pode se tornar inválido. Ainda de acordo com o autor, mesmo na falta de um experimento aleatório, deve-se hesitar em adotar estimativas que sejam sensíveis à especificação do escore de propensão. Caso a estimativa apresente sinal positivo, então o impacto é a favor do grupo de tratamento, caso contrário, o efeito é contrário.

Vale lembrar que este método calcula o ATT subtraindo-se o valor médio do resultado para os não tratados do valor estimado para os tratados. Apesar de o teste ser considerado abrangente, algumas críticas podem ser feitas em relação à possibilidade de existir um elemento não observável, que pode estar influenciando os resultados, dentro do resíduo da amostra. De acordo com Diprete e Gangl (2004), o problema pode ser resolvido com outros testes através da análise de sensibilidade. A robustez dos resultados e do método de trabalho é verificada pelo método de heterogeneidade não observada, proposto por Rosenbaum (2002).

O método identifica dois indivíduos com características observáveis, denominados de $i$ e $j$, dentro de uma distribuição logística. As razões de probabilidades para os indivíduos é dada por:

$$
\frac{\theta_{i}}{1-\theta_{i}} \text { e } \frac{\theta_{j}}{1-\theta_{j}}
$$

e o grau de associação entre eles é:

$$
\frac{\frac{\theta_{i}}{1-\theta_{i}}}{\frac{\theta_{j}}{1-\theta_{j}}}=\frac{\theta_{i}\left(1-\theta_{j}\right)}{\theta_{j}\left(1-\theta_{i}\right)}=\exp \left[\gamma\left(\mu_{i}-\mu_{j}\right)\right]
$$

11 Segundo Diprete e Gangl (2004), a metodologia empregada inicia-se com a estimativa do ATT utilizando métodos de pareamento (o escore de propensão), baseado na hipótese de independência condicional. No passo seguinte postula-se a existência de uma variável omitida $Z$, que afeta a probabilidade de participação no programa (i.e., a probabilidade de $\mathrm{D}=1$ ), condicional em $\mathrm{X}$. Conforme o impacto potencial de $\mathrm{Z}$ sobre $\mathrm{D}$ (expresso em termos dos limites das odds ratio) torna-se mais forte, o intervalo de confiança dos efeitos estimados amplia-se, e o nível de significância do teste da hipótese nula, de que não há efeito de D sobre Y, aumenta (i.e, o p-valor eleva-se). 
Se os indivíduos possuírem as mesmas características observáveis, então o vetor x se cancela. Deste modo, se não houver diferenças nas variáveis não observadas então $\mu_{i}-\mu_{j}=0$ e, ainda se estas variáveis não influenciarem os resultados $\gamma=0$, o grau de associação será igual a 1, implicando a não existência de viés de seleção. Caso contrário, existe a possibilidade de alguma característica não observável estar influenciando os resultados.

Os limites de Rosenbaum são expressos pela desigualdade:

$$
\frac{1}{e^{\gamma}} \leq \frac{\theta_{i}\left(1-\theta_{j}\right)}{\theta_{j}\left(1-\theta_{i}\right)} \leq e^{\gamma}
$$

Sendo assim, indivíduos aparentemente similares podem diferir em suas probabilidades para um valor até $e^{\gamma}=2$. Segundo Rosenbaum (2002), o valor do exponencial " $\gamma$ ” expressa a medida do grau de ruptura de uma amostra livre do viés de seleção. Assim, o método permite a expansão do intervalo de comparação das possíveis características não observáveis, até atingir o limite que a amostra suporta sem afetar os resultados das variáveis observáveis.

\subsection{Base de dados}

A base de dados será oriunda da Pesquisa Nacional por Amostra de Domicílios (PNAD) de 2011. A amostra será formada por dois grupos de trabalhadores com idade entre 60 e 80 anos: Grupo 1, formado por trabalhadores que recebem algum rendimento de aposentadoria e que exercem algum tipo de atividade econômica (Grupo tratado); Grupo 2, formado por trabalhadores que possuem as mesmas características observáveis e que exercem algum tipo de atividade econômica, mas que não recebem rendimento de aposentadoria (Grupo controle).

No referente à definição de aposentado, a PNAD (2011) considera como aposentado a pessoa que, na semana de referência da pesquisa, é jubilada, reformada ou aposentada pelo Plano de Seguridade Social da União ou por instituto de previdência social federal (Instituto Nacional do Seguro Social INSS), estadual ou municipal, inclusive pelo Fundo de Assistência e Previdência do Trabalhador Rural - FUNRURAL. Neste estudo foi considerado como aposentado àqueles indivíduos que recebe rendimentos de aposentadoria.

O INSS oferece quatro formas diferentes de aposentadoria ao trabalhador brasileiro: a) Aposentadoria especial - concedida ao trabalhador que exerce função de risco prejudicial à sua saúde ou à sua integridade física; b) Aposentadoria por idade - concedida de forma distinta, entre dois grupos, os trabalhadores urbanos e rurais; c) Aposentadoria por invalidez - concedida aos trabalhadores que por algum motivo foram considerados pela perícia da Previdência Social como impossibilitados de exercer uma profissão que gere 
renda pela força de trabalho; d) Aposentadoria por tempo de contribuição concedida aos trabalhadores que contribuíram por pelo menos 35 anos, se for homem e, por 30 anos, se for mulher. Existe, ainda, a possibilidade de o trabalhador requerer sua aposentadoria fazendo a combinação do tempo de contribuição com a idade mínima.

\section{Resultados e Discussões}

\subsection{Análise descritiva}

A amostra da pesquisa é composta por 36.001 trabalhadores ocupados, com idade entre 60 e 80 anos, sendo $22.866(63,1 \%)$ pertencente ao Grupo 1 (trabalhadores aposentados e ocupados - Grupo tratamento) e 13.135 (36,9\%) pertencente ao Grupo 2 (trabalhadores não aposentados - Grupo controle). Os dados relativos a amostra e as variáveis de análise estão descritos na Tabela 1, em valores absolutos e percentuais, em relação a cada um dos grupos citados.

A análise descritiva será feita com base nos dois grupos, de forma comparativa. Primeiramente, observa-se que o número relativo de trabalhadores ocupados é maior dentro do grupo dos não aposentados $(38,3 \%)$ comparativamente ao do grupo dos aposentados (24,5\%). A maior participação ocupacional do grupo dos não aposentados é um resultado esperado dado a maior necessidade destes trabalhadores em auferir algum rendimento. De forma similar, mas sob outra visão, a participação dos trabalhadores aposentados no mercado de trabalho, segundo Camarano (2001), Maia (2008) e Queiroz e Ramalho (2009), é explicada pela necessidade de complemento da renda da aposentadoria.

Em relação ao sexo do trabalhador, o resultado encontrado merece uma atenção especial. Embora a participação das mulheres seja maior nos dois grupos estudados, a diferença percentual observada no grupo dos aposentados é mínima, enquanto que para o grupo dos não aposentados a diferença é significativa (64,7\% contra $35,3 \%$ dos homens). Tal fato pode estar relacionado, por um lado, as maiores oportunidades de trabalho criadas para as mulheres (Scorzafave e Menezes-Filho 2005) e, por outro, a possíveis atributos não-observados que influenciariam diretamente o adiamento da aposentadoria (Queiroz et al., 2008).

Em relação à condição de chefe na família, o maior percentual está no grupo dos aposentados (67,4\% contra 57,0\% do grupo dos não aposentados). A explicação pode ser justificada pela necessidade de complemento do valor da aposentadoria, conforme Maia (2008). 
TABELA 1. ANÁLISE DESCRITIVA DA AMOSTRA DE TRABALHADORES OCUPADOS. 2011.

\begin{tabular}{|c|c|c|c|c|}
\hline \multirow[t]{2}{*}{ Variáveis } & \multicolumn{2}{|c|}{$\begin{array}{c}\text { Grupo } 1 \text { - Tratado } \\
\text { Aposentados }\end{array}$} & \multicolumn{2}{|c|}{$\begin{array}{l}\text { Grupo } 2 \text { - Controle } \\
\text { Não Aposentados }\end{array}$} \\
\hline & Abs. & $\%$ & Abs. & $\%$ \\
\hline Ocupado & $5 \cdot 595$ & 24,47 & 5.033 & 38,32 \\
\hline Chefe & $15 \cdot 377$ & 67,43 & 7.489 & 57,01 \\
\hline \multicolumn{5}{|l|}{ Sexo } \\
\hline Masculino & 11.415 & 49,92 & 4.636 & 35,30 \\
\hline Feminino & 11.451 & 50,08 & 8.499 & 64,70 \\
\hline \multicolumn{5}{|l|}{ Raça } \\
\hline Branco & 11.959 & 52,30 & 6.460 & 49,18 \\
\hline Não branco & 10.907 & 47,70 & 6.676 & 50,82 \\
\hline \multicolumn{5}{|l|}{ Faixa de idade } \\
\hline De 76 a 80 anos & 3.487 & 15,25 & 1.160 & 8,83 \\
\hline De 66 a 70 anos & 6.598 & 28,85 & 2.721 & 20,72 \\
\hline De 71 a 75 anos & 5.073 & 22,19 & 1.792 & 13,64 \\
\hline De 76 a 80 anos & 3.487 & 15,25 & 1.160 & 8,83 \\
\hline \multicolumn{5}{|c|}{ Escolaridade (anos de estudo) } \\
\hline Menos de 1 ano & 7.119 & 31,13 & $3 \cdot 717$ & 28,29 \\
\hline De 1 a 4 anos & 8.928 & 39,04 & 5.126 & 39,03 \\
\hline De 5 a 9 anos & 2.271 & 9.93 & 1.735 & 13,21 \\
\hline De 10 a 12 anos & 2.548 & 11,15 & 1.664 & 12,67 \\
\hline $\begin{array}{l}\text { Mais de } 12 \text { anos } \\
\text { Região }\end{array}$ & 2.000 & 8,75 & 893 & 6,80 \\
\hline Norte & 2.360 & 10,32 & 1.713 & 13,04 \\
\hline Nordeste & 6.873 & 30,06 & $3 \cdot 373$ & 25,68 \\
\hline Sudeste & 7.156 & 31,30 & 4.670 & 35,56 \\
\hline Sul & $4 \cdot 531$ & 19,81 & 1.964 & 14,95 \\
\hline Centro Oeste & 1.946 & 8,51 & 1.415 & 10,77 \\
\hline Zona Urbana & 18.361 & 80,30 & 12.100 & 92,12 \\
\hline Zona Rural & 4.505 & 19,70 & 1.035 & 7,88 \\
\hline Renda Média (R\$) & \multicolumn{2}{|c|}{$1.060,46$} & \multicolumn{2}{|c|}{$1.435,91$} \\
\hline Horas de Trabalho* & \multicolumn{2}{|c|}{32} & \multicolumn{2}{|c|}{40} \\
\hline Total (obs.) & \multicolumn{2}{|c|}{22.866} & \multicolumn{2}{|c|}{13.135} \\
\hline
\end{tabular}

Fonte: Elaboração própria com dados da PNAD, 2011.

* Número médio de horas de trabalho na semana de referência. 
Em relação à faixa etária, a maior concentração está entre os trabalhadores com idade entre 60 e 65 anos. Este resultado já era esperado, em virtude do próprio ciclo de vida laboral do trabalhador. É importante destacar o elevado percentual observado para esta faixa etária (entre 60 e 65 anos) no grupo dos não aposentados $(56,8 \%)$ pode estar sendo influenciado pelos trabalhadores que ainda não solicitaram (ou ainda não possuem direito) a requerer sua aposentadoria por contribuição, por exemplo.

No referente à renda média e às horas médias de trabalho, os resultados mostram que o grupo dos aposentados ocupados tem renda média ( $\mathrm{R} \$ 259,48$ contra $\mathrm{R} \$ 550,20)$ e horas médias de trabalho (2,62 contra 3,08 horas) inferior ao grupo dos não aposentados ocupados. Estes resultados podem indicar que a renda proveniente da aposentadoria permite aos trabalhadores um número menor de horas para realização do trabalho semanal, de modo que, a renda do trabalho também é menor.

Por fim, em relação às variáveis escolaridade, raça e região, os percentuais entre os dois grupos não diferem significativamente.

\subsection{Análise econométrica}

A análise econométrica do Propensity Score Matching (PSM) e o cálculo do impacto do tratamento (no caso, o recebimento dos rendimentos de aposentadoria) sobre o salário do trabalho (Average Treatment Effect on the Threat - ATT) é feita a partir do pareamento estratificado da amostra. O método de pareamento dividiu a variação dos escores de propensão em intervalos de forma que, em cada intervalo, as unidades tratadas (Grupo 1) e de controle (Grupo 2) apresentassem, em média, a mesma probabilidade de receber o tratamento (receber o rendimento de aposentadoria). O pareamento da amostra teve como base de referência as características observáveis (sexo, chefe, raça, região, faixas de idade, anos de estudo e região metropolitana) dos dois grupos estudados ${ }^{12}$.

Os resultados da estimação podem conter a existência de viés oculto na amostra. Para que o pareamento seja consistente e com a menor possiblidade da existência de vieses foram realizados testes específicos. Segundo Diprete e Gangl (2004), um resultado pode ser considerado estatisticamente significativo, com maior possibilidade de robustez do pareamento se a amostra apresentar menos de $5 \%$ de viés no pareamento. A Tabela 2 retrata o percentual de redução de viés, antes e depois do pareamento, e comprova, na coluna viés, que o pareamento ocorreu de forma satisfatória visto que em praticamente todas as variáveis a redução o valor do viés se reduziu a índices inferiores a $5 \%$, com exceção para a faixa etária dos 60 aos 65 anos que, após uma redução de $87,8 \%$, ainda permaneceu com cerca de 5,8\% de possibilidade de viés. doze anos de estudo e na idade dos setenta e seis aos oitenta anos; amostras pertencentes à região centro oeste também apresentaram colinearidade e foram excluídas. 
TABELA 2. PERCENTUAL DE REDUÇÃO DE VIÉS ANTES E DEPOIS DO PAREAMENTO - 2011.

\begin{tabular}{l|l|c|c|c|c|c}
\hline Variáveis & \multicolumn{1}{c}{ Efeito } & $\begin{array}{c}\text { Grupo 1 } \\
\text { Tratado }\end{array}$ & $\begin{array}{c}\text { Grupo 2 } \\
\text { Controle }\end{array}$ & Viés & Redução & t \\
\hline Chefe & Não pareado & 0,6725 & 0,6072 & 13,60 & - & 12,53 \\
& Pareado & 0,6724 & 0,6662 & 1,30 & 90,50 & 1,41 \\
\multirow{2}{*}{ Sexo } & Não pareado & 0,4992 & 0,3530 & 29,90 & - & 27,15 \\
& Pareado & 0,4991 & 0,4883 & 2,20 & 92,60 & 2,32 \\
\multirow{2}{*}{ Raça } & Não pareado & 0,5230 & 0,4918 & 6,20 & - & 5,70 \\
& Pareado & 0,5229 & 0,5134 & 1,90 & 69,60 & 2,03
\end{tabular}

Faixa Etária

$\begin{array}{llccccc}\text { De 6o a } & \text { Não pareado } & 0,3371 & 0,5681 & -47,70 & - & -43,86 \\ \text { 65 anos } & \text { Pareado } & 0,3372 & 0,3653 & -5,80 & 87,80 & -6,30 \\ \text { De 66 a } & \text { Não pareado } & 0,2886 & 0,2072 & 18,90 & - & 17,04 \\ \text { 70 anos } & \text { Pareado } & 0,2885 & 0,2829 & 1,30 & 93,10 & 1,32 \\ \text { De 71 a 75 } & \text { Não pareado } & 0,2219 & 0,1364 & 22,40 & - & 19,97 \\ \text { anos } & \text { Pareado } & 0,2219 & 0,2087 & 3,50 & 84,50 & 3,45 \\ \text { De 76 a } & \text { Não pareado } & 0,1525 & 0,0883 & 19,80 & - & 17,56 \\ \text { 80 anos } & \text { Pareado } & 0,1525 & 0,1432 & 2,90 & 85,60 & 2,79\end{array}$

Escolaridade

\begin{tabular}{llccccc} 
Menos de & Não pareado & 0,3113 & 0,2830 & 6,20 & - & 5,65 \\
1 ano & Pareado & 0,3114 & 0,3214 & $-2,20$ & 64,80 & $-2,30$ \\
De 1 a 4 & Não pareado & 0,3905 & 0,3903 & 0,00 & - & 0,04 \\
anos & Pareado & 0,3905 & 0,3675 & 4,70 & $-11762,50$ & 5,06 \\
De 5 a 9 & Não pareado & 0,0993 & 0,1321 & $-10,30$ & - & $-9,53$ \\
anos & Pareado & 0,0993 & 0,0960 & 1,00 & 89,80 & 1,21 \\
De 10 a 12 & Não pareado & 0,1114 & 0,1267 & $-4,70$ & - & $-4,34$ \\
anos & Pareado & 0,1115 & 0,1157 & $-1,30$ & 72,20 & $-1,43$ \\
Mais de & Não pareado & 0,0875 & 0,0680 & 7,30 & - & 6,55 \\
12 anos & Pareado & 0,0873 & 0,0994 & $-4,50$ & 38,00 & $-4,44$ \\
\hline
\end{tabular}

Fonte: Elaboração própria com dados da PNAD 2011. 
TABELA 2. PERCENTUAL DE REDUÇÃO DE VIÉS ANTES E DEPOIS DO PAREAMENTO - 2011. (CONTINUAÇÃO).

\begin{tabular}{l|l|c|c|c|c|c}
\hline Variáveis & Efeito & $\begin{array}{c}\text { Grupo 1 } \\
\text { Tratado }\end{array}$ & $\begin{array}{c}\text { Grupo 2 } \\
\text { Controle }\end{array}$ & Viés & Redução & $\mathrm{t}$ \\
\hline Região & \multicolumn{7}{c}{ Norte } & Não pareado & 0,1032 & 0,1304 & $-8,50$ & - & $-7,85$ \\
& Pareado & 0,1032 & 0,1137 & $-3,30$ & 61,60 & $-3,59$ \\
\multirow{2}{*}{ Nordeste } & Não pareado & 0,3006 & 0,2568 & 9,80 & - & 8,87 \\
& Pareado & 0,3006 & 0,2990 & 0,30 & 96,40 & 0,37 \\
\multirow{2}{*}{ Sudeste } & Não pareado & 0,3130 & 0,3555 & $-9,00$ & - & $-8,29$ \\
& Pareado & 0,3130 & 0,3105 & 0,50 & 94,10 & 0,58 \\
Sul & Não pareado & 0,1982 & 0,1495 & 12,90 & - & 11,57 \\
& Pareado & 0,1981 & 0,1899 & 2,10 & 83,40 & 2,19 \\
Centro & Não pareado & 0,0851 & 0,1077 & $-7,70$ & - & $-7,11$ \\
Oeste & Pareado & 0,0851 & 0,0869 & $-0,60$ & 92,30 & $-0,67$ \\
Zona & Não pareado & 0,8030 & 0,9212 & $-34,80$ & - & $-30,30$ \\
Urbana & Pareado & 0,8031 & 0,7915 & 3,40 & 90,20 & 3,10 \\
\hline
\end{tabular}

Fonte: Elaboração própria com dados da PNAD 2011.

Em seguida, na Tabela 3, consta o resultado do efeito médio do tratamento sobre os tratados (ATT). A análise de sensibilidade em ATT questiona o "quanto" de viés de seleção poderá estar presente a fim de tornar aceitável a hipótese nula de que não há efeito de tratamento. A análise é feita com base em três pontos: (a) a magnitude do valor estimado para o ATT; (b) seu sinal e (c) sua significância estatística.

Os resultados descritos na Tabela 3 mostram a existência de um diferencial significativo dos salários entre os grupos de tratamento (Grupo 1) e de controle (Grupo 2), favorável ao grupo de trabalhadores não aposentados (Grupo 2). Observe que a média salarial deste grupo é $\mathrm{R} \$ 629,05$ contra $\mathrm{R} \$ 259,08$ do grupo formado pelos trabalhadores aposentados. Em outras palavras, o valor médio do salário dos trabalhadores não aposentados é $\mathrm{R} \$ 369,97$ superior à média dos salários dos trabalhadores aposentados. Portanto, o rendimento de aposentadoria parece exercer um impacto negativo sobre o salário do trabalhador, indicando que o trabalhador aposentado aceita uma oferta de emprego, com salário inferior, comparativamente, ao trabalhador não aposentado. 
TABELA 3. EFEITO MÉDIO DO TRATAMENTO SOBRE OS TRATADOS (ATT). 2011.

\begin{tabular}{l|l|l|l|c|c}
\hline Variável & Amostra & $\begin{array}{r}\text { Grupo 1 } \\
\text { Tratado }\end{array}$ & $\begin{array}{l}\text { Grupo 2 } \\
\text { Controle }\end{array}$ & Diferença & Probabilidade \\
\hline \multirow{2}{*}{ Salário } & Sem pareamento & 259,48 & 550,20 & $-290,72$ & $-14,96$ \\
& ATT & 259,08 & 629,05 & $-369,97$ & $-14,09$ \\
\hline
\end{tabular}

Fonte: Elaboração própria com dados da PNAD, 2011.

Um último procedimento refere-se à análise de robustez do método que foi verificada através do teste de análise de sensibilidade proposto por Rosenbaum (2002). O procedimento separa os indivíduos com as mesmas possibilidades de pareamento a partir das características observáveis presentes em cada sujeito da amostra.

Os resultados são analisados no quadro das probabilidades com valores para o grau de ruptura, que pode variar a partir de 1 para as características que não podem ser observadas entre os trabalhadores do grupo tratado e do grupo controle. A consistência do teste pode ser verificada elevando a variação gama do grupo até alcançar uma medida que afeta o sigma dos níveis de significância.

A possiblidade da existência de ruídos (elementos não observáveis presentes na amostra) que poderiam estar comprometendo o resultado do teste inicial deve ser levada em consideração, como elementos ocultos no erro amostral. Para captar estas possíveis variações, o teste de sensibilidade permite inserir uma variação do grau de ruptura com garantia de um estudo das probabilidades livre de viés. Desse modo, variando o grau de ruptura espera-se uma variação positiva ou negativa dos níveis de significância, dada a probabilidade da presença de viés presente na amostra que não foram detectados nas variáveis observáveis.

A Tabela 4 mostra os resultados da variação gama com variação de até 10 graus. Observa-se que a ruptura ocorre no grau 8 indicando a limitação do método com a inserção de elementos não observáveis no pareamento. Os resultados do teste da análise de sensibilidade de Rosenbaum reforçam a ausência de viés em possíveis características não observáveis para um grau de liberdade de até 7, indicando, portanto, que o pareamento é robusto. Como reforça Dehejia (2005), um valor muito próximo da unidade pode ser um indicativo de viés não observável que pode influenciar o resultado das características observáveis entre os grupos. Logo, o distanciamento da unidade é um parâmetro que dá ao resultado a confiança de que o pareamento se ajusta às características observáveis e mantém-se estável para efeito do tratamento. 
TABELA 4 - ANÁLISE DE SENSIBILIDADE DOS LIMITES DE ROSENBAUM. 2011.

\begin{tabular}{c|ccc|c|c|c}
\hline \multirow{2}{*}{ Gama } & \multicolumn{3}{|c|}{ Sigma } & \multicolumn{2}{c|}{ Estimador } & \multicolumn{2}{c}{ Intervalo } \\
\cline { 2 - 7 } & Positivo & Negativo & Superior & Inferior & Superior & Inferior \\
\hline 1 & 0 & 0 & $-601,13$ & $-601,13$ & $-604,57$ & $-597,90$ \\
2 & 0 & 0 & $-654,79$ & $-476,29$ & $-656,68$ & $-471,43$ \\
3 & 0 & 0 & $-678,02$ & $-411,02$ & $-679,77$ & $-404,64$ \\
4 & 0 & 0 & $-693,33$ & $-359,40$ & $-695,37$ & $-350,18$ \\
5 & 0 & 0 & $-708,61$ & $-279,31$ & $-711,25$ & $-260,94$ \\
6 & 0 & 0 & $-718,73$ & $-205,75$ & $-720,58$ & $-190,81$ \\
7 & 0 & 0 & $-724,37$ & $-147,31$ & $-725,64$ & $-127,85$ \\
8 & 0 & 0,00000003 & $-728,07$ & $-845,29$ & $-729,35$ & $-57,97$ \\
9 & 0 & 0,212773000 & $-731,09$ & $-143,96$ & $-732,31$ & 194,37 \\
10 & 0 & 0,999532000 & $-733,79$ & 505,09 & $-734,94$ & 824,98 \\
\hline
\end{tabular}

Fonte: Elaboração própria com dados da PNAD, 2011.

\section{Considerações Finais}

Este artigo teve por objetivo comparar, através de atributos observáveis, o rendimento de dois grupos de trabalhadores, com idade entre 60 e 80 anos, no mercado de trabalho brasileiro. Para tal, fez-se uso de um método probabilístico que compara características observáveis comuns - Propensity Score Matching (PSM).

Conforme citado no artigo, o PSM é conhecido como uma técnica de pareamento e baseia-se na probabilidade dos grupos (indivíduos) apresentarem características semelhantes. Por isso, algumas críticas podem ser feitas em relação à possibilidade de existir um elemento não observável que pode influenciar o resultado na forma de um resíduo da amostra. Outra questão importante reporta-se a possível endogeneidade entre as variáveis de tratamento (rendimento de aposentadoria) e o output (salário no mercado de trabalho). A literatura econométrica retrata a dificuldade em minimizar os efeitos do viés causado por uma possível relação entre as variáveis, com alguns estudos sugerindo a possibilidade de uma modelagem com uso de Variáveis Instrumentais (IV); enquanto outros, como Nadal e Martins (2009), acreditam que o método de Propensity Matching Score é mais eficiente comparativamente à técnica de estimação de Variáveis Instrumentais (doravante VI) já que, além de corrigir o problema de endogeneidade da variável de tratamento, não exige o uso de instrumentos cuja eficácia e validades possam ser duvidosas, dificultando o processo de estimação. 
Dada estas discussões metodológicas, os resultados encontrados no estudo indicam que o impacto dos rendimentos de aposentadoria interfere negativamente no valor da renda do trabalho. Ao mesmo tempo em que se constitui uma espécie de reserva de rendimento, os benefícios da aposentadoria faz com que o trabalhador aposentado se insira no mercado de trabalho aceitando um salário mais baixo, fazendo com que a média salarial do seu grupo etário (trabalhadores com mais de 60 anos) seja reduzida para valores inferiores ao salário mínimo. Os resultados mostraram que o recebimento do benefício oriundo da aposentadoria pode provocar uma redução nos rendimentos do trabalhador da ordem de 50\% do salário de referência de um trabalhador não aposentado.

É importante deixar claro, contudo, que não se pretende, com isso, negar a importância da renda proveniente da aposentadoria na composição da renda familiar e na redução da pobreza, mas sim, apontá-la como um possível elemento responsável por esta diferença salarial entre os trabalhadores. Assim, como argumenta Damasceno e Cunha (2008), a redução no salário do trabalhador aposentado pode estar relacionada a segurança de um salário de reserva garantida pelo valor da aposentadoria ou apenas pela necessidade de sentir-se produtivo. Dessa forma, o retorno do trabalhador aposentado ao mercado de trabalho pode influenciar a renda do trabalho dos indivíduos com as mesmas características e que ainda não se aposentaram.

Por fim, é importante destacar a contribuição do estudo para a discussão acerca do comportamento do trabalhador aposentado ocupado no mercado de trabalho em relação ao seu salário. Os resultados aqui observados podem estar indicando para uma das duas vertentes citadas a seguir: 1 . Parecem ir de encontro à hipótese de que quanto maior for o montante da renda oriunda do não trabalho (por exemplo, o rendimento de aposentadoria) maior seria $o$ seu salário de reserva, logo, maiores seriam os salários recebidos, ou 2. Podem estar sugerindo que o rendimento recebido da aposentadoria não é suficiente para suprir as necessidades do trabalhador. Neste caso, o trabalhador permanece (ou retorna) ao mercado de trabalhado, aceitando uma condição salarial inferior comparativamente àqueles que estão ocupados, mas que não recebem o benefício da aposentadoria.

\section{Referências Bibliográficas}

BIRD (Banco Internacional para a Reconstrução e o Desenvolvimento) (2011). Envelhecendo em um Brasil mais Velho. Banco Mundial. Washington, D.C.

BLAU, D. M. (1994). "Labor force dynamics of older men”. Econometrica, v. 62 (1), pp. 117-156.

BORJAS, G. J. (2012). Labor Economics. Mc Graw-Hill, 6ª Ed. New York. 
BRASIL. MINISTÉRIO DA PREVIDÊNCIA SOCIAL (2011). Anuário Estatístico da Previdência Social: Suplemento Histórico (1980 a 2011). Brasília.

CAMARANO, A. A. (2001). "O idoso brasileiro no mercado de trabalho." Texto para discussão ${ }^{\circ}$ 830, IPEA.

CARRERA-FERNANDEZ, J.; MENEZES, W.F. (2001). "O Idoso no Mercado de Trabalho: Uma Análise a Partir da Região Metropolitana de Salvador”. Revista Econômica do Nordeste, v. 32 (1), pp. 52-67.

COMA, M. R. (2012).“Técnicas de evaluación de impacto: propensity score matching y aplicaciones prácticas con stata”. Instituto de Estudios Fiscales, doc. $\mathrm{n}^{\mathrm{O}} 2 / 2012$. Madri.

DAMASCENO, F. S.; CUNHA, M. S. (2008). Determinantes da participação do idoso no mercado de trabalho brasileiro. Apresentado no XI Encontro Regional de Economia - ANPEC-Sul. Área 2.

DEHEJIA, R. (2005). "Practical propensity score matching: a reply to Smith and Todd”. Journal of Econometrics, v.125 (1-2), pp. 355-364.

DIPRETE, T.; GANGL, M. (2004). "Assessing bias in the estimation of causal effects: Rosenbaum bounds on matching estimators and instrumental variables estimation with imperfect instruments". Sociological Methodology, v.34 (1), pp.271-310.

FURTADO, A. (2005). A participação do idoso no mercado de trabalho. Consultoria Legislativa. Câmara dos Deputados. Brasília.

GASPARINI, L.; ALEJO, J.; HAIMOVICH, F.; OLIVIERI, S.; TORNAROLLI, L. (2007). "Poverty among the elderly in Latin America and the Caribbean". Background paper for the World Economic and Social Survey 2007: The World Ageing Situation. Universidad de la Plata.

HOFFMANN, R. (2010). "Como aposentadorias e pensões afetam a educação e o trabalho de jovens do domicílio.” Revista Economia e Sociedade, v. 19 (38), pp. 201-209. Campinas.

IBGE - Instituto Brasileiro de Geografia e Estatística. Censo Demográfico/ 201O. URL [on-line]: <http://www.ibge.gov.br/home/estatistica/populacao/ censo2010/default.shtm>. Acesso em: 10 jul. 2013.

INSS - Instituto Nacional de Seguridade Social (2013). Boletim Estatístico da Previdência Social - BEPS. Ministério da Previdência Social, v. 18(3).

KON, A. (2006). "Mudanças recentes no perfil da distribuição ocupacional da população brasileira”. Revista brasileira de estudos de população, v.23, pp. 247-267.

LIBERATO, V.C. (2003). “A oferta de trabalho masculina pós-aposentadoria”. Brasil Urbano - 1981/2001. 78 f. Dissertação de Mestrado em Demografia. Belo Horizonte, Universidade Federal de Minas Gerais.

MAIA, A.G.; OLIVEIRA, M. M.; BALLINI, R.; DEDECCA, C. S. (2008). "Impacto dos rendimentos de aposentadoria e pensão na redução da pobreza rural”. SOBER - GT, v. 11.

MENEZES-FILHO, N. (org) (2012). Avaliação econômica de projetos sociais. Fundação Itaú. Dinâmica Gráfica e Editora. São Paulo. 
MOURA, C. S.; CUNHA, M. S. (2010) "Fatores determinantes da participação e do rendimento do idoso e não-idoso no mercado de trabalho brasileiro". A Economia em Revista - AERE, v.18(2).

NADAL, R., MARTINS, R. (2009) "Efeitos de mudanças liberalizantes da conta de capital sobre o crescimento: uma abordagem de avaliação de políticas públicas”. XXXVII Encontro Nacional de Economia (ANPEC). Foz do Iguaçu, PR.

OLIVEIRA, F. E. B.; BELTRÃO, K. I.; FERREIRA, M. G. (1997). "Reforma da Previdência”. Texto para Discussão 508. Rio de Janeiro: IPEA.

OPAS (Organização Pan-Americana da Saúde). (2005). "Envelhecimento ativo: uma política de saúde”. Título original inglês: Active ageing: a policy framework. WHO/NMH/NPH, tradução de Suzana Gontijo. Organização Pan-Americana da Saúde, 6op. Brasília.

PENCAVEL, J. (1986). “Labor supply of men: A survey”. In: ASHENFELTER, O.; LAYARD, R. (Eds). The Handbook of Labor Economics. Elsevier, v. 1. Amsterdam.

PÉREZ, E. R.; WAJNMAN, S.; OLIVEIRA, A. M. H. C. (2006). “Análise dos determinantes da participaçãono mercado de trabalho dos idosos em São Paulo”. Revista Brasileira de Estudos de População. São Paulo, v. 23 (2), pp. 269-286.

PNAD (Pesquisa Nacional por Amostra de Domicílios) (2011). URL [on-line]: <http://www.ibge.gov.br/home/estatistica/populacao/censo2010/default. shtm>. Acesso em: 10 jul. de 2013.

QUEIROZ, V. S.; RAMALHO, H. M. (2009). “A Escolha Ocupacional dos Idosos no Mercado de Trabalho: Evidências para o Brasil”. Revista Economia.

REIS, P. R. C. (2012). “Política Pública de Previdência Social e Nível de Bem Estar: Impacto sobre as famílias e municípios do Minas Gerais”. Magister Scientiae. Viçosa, Universidade Federal de Viçosa.

ROSENBAUM, P. (2002). “Observational Studies”. Springer, New York.

ROSENBAUM, P.; RUBIN D.B. (1983). "The Central role of the propensity score in observational studies for causal effects". Biometrika, v.70 (1), pp.41-55.

SCORZAFAVE, L. G.; MENEZES-FILHO, N. A. (2001). "Participação feminina no mercado de trabalho brasileiro: evolução e determinantes". Pesquisa e Planejamento Econômico, v. 31 (3), pp. 441-478.

TRUELSEN, T.; BONITA, R.; JAMROZIK, K. (2001). "Survillance of stroke: a global perspective”. International Journal of Epidemiology, v. 30, s11-s12.

WAJNMAN, S.; OLIVEIRA, A. M. H. C.;OLIVEIRA, E. L (2004). “Os Idosos no Mercado de Trabalho: Tendências e Consequências”. URL: [on-line]: <https:// ipea.gov.br/agencia/images/stories/PDFs/.../Arq_23_Cap_14.pdf $>$. Acesso em: 20 ago. de 2013.

Recebido em: 11 de setembro de 2013 Aceito em: 19 de maio de 2014 